\title{
Filosofía teatral: lecturas posibles de Deleuze ${ }^{1}$
}

\author{
Adán Salinas Araya ${ }^{2}$
}

Recibido: 26 de junio de 2019 - Aceptado: 24 de agosto de 2019

\begin{abstract}
Resumen
El artículo se detiene en algunas facetas de una posible filosofía teatral en Deleuze. Esta filosofía teatral podría ser una de las claves de interpretación para el análisis de algunas de las principales categorías del autor. Como un modo inicial de aproximación, el artículo expone tres operaciones o tropos de esta forma de filosofía: utilizar los conceptos dentro de una puesta en escena, encarnar el acontecimiento y devenir menor, tropos que pueden ser entendidos, según las palabras de Artaud, como un lenguaje a medio camino entre el gesto y el pensamiento.
\end{abstract}

Palabras clave: Deleuze - teatro - Artaud - filosofía teatral - devenir minoritario

\section{Theater philosophy: possible readings of Deleuze}

\begin{abstract}
Summary
The article sets out to consider certain facets of a possible theater philosophy in Deleuze. This theater philosophy could be one of the interpretation keys for analysis of some of the author's principal categories. As an initial means of approach, the article addresses three operations or tropes of this form of philosophy: using concepts within a staging, embodying the happening, and the lesser path; tropes that can be understood, in the words of Artaud, as a language halfway between gesture and thought.
\end{abstract}

Keywords: Deleuze - theater - Artaud - theater philosophy - lesser path

1 El presente artículo reelabora algunas ideas expuestas en la ponencia presentada en Jornadas Gilles Deleuze. Filosofía, Artes, Política, en el CEA de la Universidad de Playa Ancha, en 2015. El artículo forma parte del proyecto postdoctoral FONDECYT No 3170031.

2 Doctor en Filosofía por la Universidad Complutense de Madrid. Académico de la Universidad Academia de Humanismo Cristiano. Actualmente dirige el Doctorado en Estudios Transdisciplinares Latinoamericanos (DETLA) en dicha Casa de Estudios y es profesor colaborador del Magíster en Filosofía Política en la Universidad de Santiago de Chile. Contacto: asalinasa@docentes.academia.cl

ORCID: https://orcid.org/0000-0002-0974-284X 


\title{
Filosofia teatral: possíveis leituras de Deleuze
}

\begin{abstract}
Resumo
O artigo foca em algumas faces de uma possível filosofia teatral em Deleuze. Esta filosofia teatral poderia ser uma das chaves de interpretação para a análise de algumas das principais categorias do autor. Como um modo inicial de aproximação, o artigo expõe três operações ou tropos desta forma de filosofia: utilizar os conceitos dentro de uma encenação, encarnar o acontecimento e devir menor, tropos que podem ser entendidos, segundo as palavras de Artaud, como "uma linguagem a meio caminho entre o gesto e o pensamento".
\end{abstract}

Palavras-chave: Deleuze - teatro - Artaud - filosofia teatral - devir minoritário

\section{Introducción}

A continuación, se presentan algunos tópicos para la comprensión de la filosofía deleuziana como una filosofía teatral. Se busca, en primer lugar, acercarse a la idea de una filosofía teatral y destacar tres tropos de ella: encarnar el acontecimiento, producir el devenir menor y utilizar los conceptos dentro de una puesta en escena. Se toman elementos de algunos de los textos más importantes de Deleuze, como Mil Mesetas, escrito junto a Guattari, y Diferencia y Repetición, y también se recogen textos que, a los cánones interpretativos tradicionales, pueden parecer textos menores, sabiendo que, en la filosofía deleuziana, el devenir menor es un asunto de la mayor importancia. Dialogan con Deleuze otros personajes conceptuales expresados en forma de autor, en particular Antonin Artaud, Carmelo Bene y Joe Bousquett. El artículo debe ser concebido, entonces, como una propuesta exploratoria que puede ser complementada, tanto con la aproximación a otros textos de Deleuze sobre aspectos teatrales, como también mediante la exposición de algunos otros tropos que puedan complementar la exposición inicial de lo que aquí se concibe como encarnar el acontecimiento, producir el devenir menor y utilizar los conceptos dentro de una puesta en escena. 


\section{Puesta en escena}

La filosofía no como pensamiento, sino como teatro: teatro de mimos con escenas múltiples, fugitivas e instantáneas donde los gestos, sin verse, se hacen señales: teatro donde, bajo la máscara de Sócrates, estalla de súbito el reír del sofista; donde los modos de Spinoza dirigen un anillo descentrado mientras que la substancia gira a su alrededor como un planeta loco; donde Fichte cojo anuncia «yo fisurado/yo disuelto»; donde Leibniz, llegado a la cima de la pirámide, distingue en la oscuridad que la música celeste es el Pierrot lunar. En la garita de Luxembourg, Duns Scoto pasa la cabeza por el anteojo circular; lleva unos considerables bigotes; son los de Nietzsche disfrazado de Klossovski (Foucault 1995 47).

Es un hecho conocido que Foucault tituló su ensayo sobre Deleuze como Theatrum Philosophicum. Ahora bien, este hecho, aunque conocido, suele pasar sin mayor comentario. La perspectiva de trabajo de Deleuze, al menos en parte importante, puede entenderse bajo la figura de una "filosofía teatral". Me interesa esbozar aquí un acercamiento a esta idea de una filosofía teatral y no tanto un itinerario crítico de los dichos de Deleuze sobre el teatro, tarea interesante y de la que hay algunos antecedentes valiosos (Sarda 2006 Chevallier 2011 2014 Garcin-Marrou 2011), pero que tiene una intención diferente. Propongo entonces comprender la filosofía deleuziana como puesta en escena, con la diversidad de elementos que una puesta en escena tiene y que, en última instancia, implica una experiencia o es una forma de relación consigo mismo. Como primer elemento, esta filosofía teatral ocupa el concepto hasta cierto punto, y hasta otro cierto punto lucha con el concepto. Mucho se ha comentado la famosa propuesta de ¿Qué es la filosofía?, según la cual Filosofía es crear conceptos (Deleuze y Guattari 2000); y, sin embargo, la noción de "concepto" que Deleuze propone es altamente problemática. En Diferencia y Repetición, Deleuze asalta la noción de "concepto" de Hegel como una herramienta de la lógica de la representación. 
Hegel sustituye la verdadera relación de lo singular y de lo universal en la Idea por la relación abstracta entre lo particular y el concepto en general. Se limita, pues, al elemento reflejado de la «representación», a la simple generalidad. Representa conceptos en lugar de dramatizar Ideas: hace un falso teatro, un falso drama, un falso movimiento (Deleuze 2002 34).

Es Ilamativo, entonces, que Deleuze y Guattari posteriormente definan la filosofía como "la disciplina que consiste en crear conceptos" (2000 11). Me parece que esta contradicción inicial muestra que la noción de "concepto", que Deleuze propone como la actividad definitoria de la filosofía, es una forma particular del concepto, una noción problematizada de "concepto" que no es la noción hegeliana, ni tampoco otra noción. El propio Deleuze sugería algunas pistas al declarar que este tipo de conceptos no refiere a esencias, sino a acontecimientos.

La filosofía se ha ocupado siempre de conceptos, y hacer filosofía es intentar crear o inventar conceptos. Pero hay varios aspectos posibles en los conceptos. Durante mucho tiempo, los conceptos han sido utilizados para determinar lo que una cosa es (esencia). Por el contrario, a nosotros nos interesan las circunstancias de las cosas - ¿en qué caso? ¿dónde y cuándo? ¿cómo?, etc.-. Para nosotros, el concepto debe decir el acontecimiento, no la esencia (Deleuze 1995 45).

Pero la clave de lectura más decisiva me parece que es el teatro, o la filosofía como teatro. La crítica anterior ya sugiere esto: Hegel representa conceptos en vez de dramatizar ideas. Se trata de un falso teatro, un teatro de la representación o del concepto, y no un teatro del dramatizar o de la idea o del movimiento. Deleuze, en una conferencia de 1967, anterior incluso a Diferencia y Repetición, titulada sugerentemente «El método de dramatización» (Deleuze 2005), presenta por primera vez esta preocupación: ¿en qué caso?, ¿dónde?, ¿cómo?, incluso ¿por quién? Se trata del tipo de preguntas que, en el método de la dramatización, sustituyen a la pregunta platónica por la esencia: "¿qué es?". Por otra parte, el problema de la representación, 
aquí mencionado, coincide con lo que plantea sobre la representación y el poder, en el ensayo que escribe acerca del teatro de Carmelo Bene (Deleuze 2003). Especialmente en la última parte del ensayo, Deleuze esboza una visión del teatro en la que se opone al poder, plasmación estática, y en cambio hace juego con una potencia siempre en movimiento que en tal ensayo se centra en el devenir minoritario. Sobre el teatro como actividad artística $-\mathrm{y}$ no solo sobre el teatro de Bene- afirmará: "Esta función anti-representativa sería trazar, constituir de alguna manera una figura de la conciencia minoritaria, como potencialidad de cada uno. Convertir en presente y virtual, una potencialidad es algo completamente diferente a representar un conflicto" (Deleuze 2003 98-99). Pienso, entonces, que cuando Deleuze y Guattari hablan de conceptos en ¿Qué es la filosofía?, habría que entender que se trata de conceptos de otro tipo y no los conceptos de una filosofía de la representación. Cada concepto va acompañado de un conjunto, una serie o una multiplicidad de elementos sin los cuales no podría explicarse y que, en cierto sentido, responden a las preguntas de la dramatización; pues "Todo concepto remite a un problema, a unos problemas sin los cuales carecería de sentido, y que a su vez solo pueden ser despejados y comprendidos a medida que se vayan solucionando" (Deleuze y Guattari 2000 22). Pero, además, los conceptos estarían acompañados no solo por estas constelaciones que desarrollan su contexto de problematización, sino por personajes conceptuales, y aquí vemos un paso más. El primer ejemplo que Deleuze propone es el de amigo.

Los conceptos, ya lo veremos, necesitan personajes conceptuales que contribuyan a definirlos. Amigo es un personaje de esta índole, del que se dice incluso que aboga por unos orígenes griegos de la filo-sofía: las demás civilizaciones tenían Sabios, pero los griegos presentan a esos «amigos» (Deleuze y Guattari 2000 8).

Amigo es el personaje conceptual detrás de la philia filosófica, el personaje que, de hecho, hace operativa la posibilidad del filósofo como "amigo de la sabiduría". También el cogito cartesiano tendría su personaje conceptual en el pensador privado. Permítanme que me entretenga 
un momento con estos personajes, cito: "...el pensador privado forma un concepto con unas fuerzas innatas que todo el mundo posee por derecho por su cuenta (yo pienso). Nos encontramos aquí con un tipo de personaje muy extraño, que quiere pensar y que piensa por sí mismo, por la «luz natural»" (Deleuze y Guattari 2000 63). En el mismo análisis, Sócrates aparece como el personaje conceptual de los diálogos platóni$\cos (65)$ y, en otros análisis, Dionisios, personaje de Nietzsche, el idiota de Nicolás de Cusa, o el caballero de la fe y el burgués endomingado de Kierkegaard (Deleuze 2002 72), todos ellos personajes conceptuales. En algunas ocasiones estos personajes están en relación de antagonismo con otros personajes al interior de una misma escena filosófica, de hecho, el amigo o amante de la sabiduría debe competir-combatir con el pretendiente o falso sabio encarnado por el sofista, quien será también antagonista de Sócrates, junto al ignorante inconsciente. Dionisios, por su parte, será amigo de Zaratustra, quien a veces se confunde con el Anticristo, o bien pueden ser máscaras que se superponen sobre un mismo personaje: "Recordemos la canción de Ariadna, puesta en boca del viejo Encantador: aquí, se superponen dos máscaras: la de la mujer joven, casi la de una Koré, se aplica sobre la de un anciano repugnante. El actor debe interpretar el papel de un anciano que interpreta a su vez el de la Koré" (Deleuze 2002 33). Este personaje conceptual se vuelve tan identificable que se podría hablar, por ejemplo, del Dionisios de Nietzsche, que en efecto no es el Dionisios mitológico o mistagógico.

El destino del filósofo es convertirse en su o sus personajes conceptuales, al mismo tiempo que estos personajes se convierten ellos mismos en algo distinto de lo que son históricamente, mitológicamente o corrientemente (así tenemos el Sócrates de Platón, el Dionisios de Nietzsche, el Idiota de Cusa). El personaje conceptual es el devenir o el sujeto de una filosofía, que asume el valor del filósofo, de modo que Cusa, o incluso Descartes, deberían firmar «el Idiota», de la misma forma que Nietzsche «el Anticristo» o «Dionisio contra el crucificado» (Deleuze y Guattari 2000 66).

Nuevamente hay una serie de acercamientos a lo que planteaba sobre el teatro de Carmelo Bene, la desaparición de Romeo, en su versión de Romeo y Julieta, crea un nuevo Mercuzio, que no estaba 
presente en el original (Deleuze 2003 77). Es el Mercuzio de Bene y ningún otro.

Se trata, entonces, de una puesta en escena. Esta es la modalidad básica de la filosofía. Ello no quiere decir que los personajes conceptuales no existan, o equivalgan a símbolos o figuraciones. "EI personaje conceptual no tiene nada que ver con una personificación abstracta, con un símbolo o una alegoría, pues vive, insiste" (Deleuze 2000 66). Ahora bien, esta forma de entender la filosofía, a mi juicio, marca la manera de acercarse a los conceptos del propio Deleuze. De este modo entiendo su rechazo a la metáfora, la insistencia en que sus propios conceptos y sus personajes conceptuales no son metáforas, o alegorías, o símbolos. Por ejemplo, Un cuerpo sin órganos, como horizonte de producción de un sujeto (o habría que decir, en rigor, como un modo de individuación precisamente opuesto al sujeto) y como personaje conceptual de una escena de resistencia y desestratificación del organismo político, no es una metáfora, sino parte de una puesta en escena, de hecho, parte de las indicaciones concretas que el director de escena entrega para captar la comprensión de un personaje. Sucede algo similar con una rostridad que forma un modo de individuación que deshace el rostro. Lo que Deleuze propone respecto de Nietzsche puede aplicársele a sí mismo.

Cuando Nietzsche dice que el superhombre se parece más a Borgia que a Parsifal, cuando sugiere que participa a la vez de la orden jesuítica y del cuerpo de oficiales prusianos, solo se llega a la comprensión de estos textos tomándolos por lo que realmente son: observaciones de director teatral que indican cómo debe ser «interpretado» el superhombre (Deleuze 2002 33).

Esta concepción filosófica es la que impulsa a Deleuze a componer algo así como una novela psicoanalítica, o a colectar a Kafka y Proust, al mismo tiempo que a Spinoza. Especialmente, según me parece, es lo que impulsa a hacer coincidir a Kierkegaard y Nietzsche como parte de una forma de filosofía, de una filosofía del porvenir. 
En este sentido hay algo completamente nuevo que comienza con Kierkegaard y Nietzsche. Ya no reflexionan sobre el teatro a la manera hegeliana. Tampoco hacen un teatro filosófico. Inventan, en la filosofía, un equivalente increíble del teatro, y con ello, fundan ese teatro del porvenir, al mismo tiempo que una filosofía nueva (Deleuze 2002 32).

Se trata de una forma de filosofía que Deleuze no ha inventado, el teatro es, hasta cierto punto, similar a la literatura, pero es también diferente, pues está definido por el movimiento. Habría una filosofía y "su otro", utilizando la expresión que rescata GarcinMarrou (2011); o a la inversa —utilizando la expresión de Artaud (1978), que luego enfatiza Klossowski (1981), también en un ensayo sobre el teatro de Carmelo Bene-: habría un teatro y su doble, (una filosofía) de la representación y un teatro y su doble (una filosofía) de la repetición.

El teatro de la repetición se opone al teatro de la representación, así como el movimiento se opone al concepto y a la representación que lo relaciona con el concepto. En el teatro de la repetición se experimentan fuerzas puras, trazos dinámicos en el espacio que actúan sobre el espíritu sin intermediarios, y que lo unen directamente a la naturaleza y a la historia, un lenguaje que habla antes que las palabras, gestos que se elaboran antes que los cuerpos organizados, máscaras previas a los cuerpos, espectros y fantasmas anteriores a los personajes — todo el aparato de la repetición como «potencia terrible» (Deleuze 2002 34-35).

\section{Encarnar el acontecimiento}

En Lógica del Sentido, Deleuze presta una atención especial al actor. Es el actor quien puede romper el tiempo circular que retorna, y en él se expresa el acontecimiento. Por eso puede quebrar también la lógica de la representación, la imagen de una filosofía de lo uno y 
de la identidad. Le corresponde entonces un papel primordial en la filosofía teatral que antagoniza con la filosofía de la representación.

El actor permanece en el instante, mientras que el personaje que interpreta espera o teme en el porvenir, se acuerda o se arrepiente en el pasado: es en este sentido que el actor representa. Hacer corresponder el mínimo de tiempo interpretable en el instante con el máximo de tiempo pensable según el Aión. Limitar la efectuación del acontecimiento a un presente sin mezcla, hacer el instante tanto más tenso e intenso, tanto más instantáneo cuanto que representa un futuro y un pasado ilimitados, éste es el uso de la representación: el mimo, ya no el adivino (Deleuze 2005b 181).

Tenemos de una parte al actor y de otra al personaje. El actor puede ser un contra-dios, precisamente por hacerse resonar en diversos personajes, por la capacidad de usar una máscara sobre la máscara y porque en él se realiza la contraefectuación del acontecimiento, es decir, se realiza al mismo tiempo que se escapa. Lo que interpreta el actor, nos dice Deleuze, es un tema que se constituye por los componentes del acontecimiento, es decir, se compone de las singularidades comunicativas liberadas de los límites de los individuos.

El actor efectúa el acontecimiento, pero lo hace de una manera muy particular, lo efectúa en el sentido último de la contraefectuación, es decir, efectúa el acontecimiento desdoblando esa efectuación y lo que podemos ver de esto es solo el contorno, el esplendor del acontecimiento.

El actor puede jugar, ensaya una y otra vez sus escenas, de alguna manera debe hacerlas personales. En el actor hay un sutil juego. Lo que el actor dramatiza no puede romper al actor, el disfraz no puede parecer disfraz, no habría nada más patético que darse cuenta que una actuación resultara falsa. No puede ser falsa, pero no puede ser verdadera. Y aquí hay una paradoja interesante. Esta prioridad del actor en todo caso debe ponerse en suspenso, su capacidad de dramatizar no es absoluta. En cierto sentido es una superficie de contacto entre una carne (un modo de individuación o un sujeto) y un personaje 
conceptual, no hay que olvidar, se trata siempre de un juego de tres términos respecto a los modos de individuación; en este caso: una carne (un yo-uno o una multiplicidad subjetiva), un actor, un personaje.

\section{Rostridad. Devenir menor}

El rostro es la expresión del sujeto. Un sujeto tiene un rostro; por el contrario, un yo-multiplicidad tiene una infinidad de rostros, tantos como intensidades subjetivas lo recorren.

Es importante recalcar que aquí hay en juego una noción específica de sujeto, subjetividad o modo de individuación. No se trata de un sujeto entendido como el resultado de unas ciertas relaciones por las cuales se está atado a otro o a cierta conciencia de uno mismo. Que serían los dos sentidos que exploró Foucault sobre la subjetivación (Foucault 1988 231s), como sujeción a otro y como atadura a una cierta conciencia de sí. Como contraposición, "subjetivación" se puede entender también como afirmación del sujeto, como contrafuerza y resistencia de las relaciones de dominación, un sujeto que se construye en esas resistencias y, hasta cierto punto, las rompe o tiene como vocación romperlas. Se trata del sujeto entendido como autonomía y emancipación del yo o, también y preferentemente, de un sujeto colectivo. Me parece, por ejemplo, que esta es la noción de "subjetivación" que utiliza Rancière (2000, 146ss).

Aquí hay dos nociones diferentes de "sujeto" — hasta cierto punto incluso en contradicción-, ambas muy importantes en la teoría política contemporánea. Mientras Foucault enfatiza la sujeción, Rancière entiende al sujeto como emancipación. Por supuesto, habría que matizar, habría que decir que en Foucault, además de los procesos de sujeción, son posibles las alternativas de subjetividad, que son también resistencias, aunque más claramente individuales que colectivas. Anótese entonces el matiz. Me interesa enfatizar que, a estas dos nociones diferentes de "subjetivación", habría que sumar la noción que pone en juego Deleuze y que no es completamente similar a ninguna de estas dos nociones anteriores. En Deleuze hay una tensión entre el 
sujeto y otros modos de individuación, está tensión se expresa en la contraposición entre organismo y cuerpo sin órganos; y también entre rostro y rostridad. El rostro apuntaría precisamente a la formación de un sujeto, mientras que la rostridad apuntaría a un modo de individuación diferente y múltiple. Me parece que aquí la crítica al rostro es una crítica al sujeto identitario, al yo neurótico. Deleuze no busca al sujeto sino a las intensidades subjetivas (Landaeta y García 2007) que tienden a modos de individuación que dejan atrás al yo. Para Deleuze, la subjetivación es inscripción en la lógica identitaria del yo, lógica a la que contribuye el psicoanálisis con la imposición de la estructura neurótica como matriz de interpretación (Salinas 2003). Por esta misma razón, hay que entender el rostro como entendemos el cuerpo, no como una especialidad biológica, sino también estética, política, mística, ética.

La significancia es inseparable de una pared blanca sobre la que inscribe sus signos y sus redundancias. Y la subjetivación es inseparable de un agujero negro en el que sitúa su conciencia, su pasión, sus redundancias... Un rostro es algo muy singular: sistema pared blanca-agujero negro (Deleuze y Guattari 2004 173).

El rostro es capaz de un lenguaje y de la falsedad; por tanto, es capaz de significancia; hace de conexión entre la carne, la significación y la subjetivación. La cabeza está en el organismo, pero el rostro no; una cabeza, aunque sea humana, no constituye de suyo un rostro.

En tal sentido, el rostro es una sobrecodificación o desterritorialización absoluta, es pasar de un estrato a otro; vale decir, el rostro deja de ser parte de la cabeza, del organismo, y comienza a ser parte de la significancia y la subjetivación, en la medida en que expresa un lenguaje y que permite identificar a un sujeto. El rostro ya no es parte del organismo, se ha desterritorializado, pero tal desterritorialización lo ha llevado a otro estrato, el rostro se encuentra en un metaestrato respecto del organismo, no en cuanto la significación o la subjetivación sean un estrato superior, sino en cuanto lo son para el rostro. Lo mismo podría pasar con un par de cicatrices, tan características, tan 
conocidas, que sirvan para identificar un sujeto: "no he visto nunca esa cara, pero esas cicatrices las conozco". Juego de la identificación. O también con una mano, un tatuaje, una marca, la pérdida de un par de dedos, una deformación; todo ello puede transformar una mano en un rostro, al menos en el ámbito de la subjetivación. Usualmente las manos están más rostrificadas, en cuanto expresan, conjuntamente con el rostro, un lenguaje común, pero en conexión con él.

Si la rostrificación arranca la cabeza del organismo hacia un metaestrato, la rostrificación puede ser un primer paso a la constitución de un modo de individuación. Comenzar a hacer de todo el cuerpo un rostro es sacarlo del estrato del organismo, aunque es solo el primer paso: queda por formar una rostridad.

Se trata de la experiencia actoral. El ropaje, el disfraz, van convirtiendo al organismo en rostro; los órganos dejan de ser tales: comienzan a constituirse en un mapa. Aunque todavía se está atado a la significancia, a los colores, a los valores, a los cortes y a la subjetivación. Es necesario llegar a ser no un rostro, sino una rostridad, una multiplicidad de rostridades, de disfraces de expresiones que rompen al rostro. Dejar de ser un organismo y comenzar a ser un rostro constituye un paso, aunque no el paso definitivo, pues todavía se encuentra en la significancia y la subjetividad. Dejar de ser un rostro y constituirse en una rostridad constituye un paso más. Claro que una rostridad no es una máscara o un personaje, sino una forma de individuación. Hay aquí una serie, o un juego de tres términos: modo de individuación, actor, personaje. De esto se trata en definitiva el problema de los modos de individuación sometido al ejercicio teatral. "El rostro es una política" (Deleuze y Guattari 2004 186). La política del rostro, entonces, es la política del sujeto identitario, del que puede decir "yo", afirmarse como un yo. La experiencia actoral trastoca precisamente la experiencia del sujeto y ayuda a instalar modos de individuación diferentes.

El rostro se produce en una serie. Primero un agenciamiento maquínico de poder (agenciamiento concreto y autoritario), luego una máquina abstracta de rostridad (pared blanca-agujero negro, o significancia y subjetivación); por último, el establecimiento de una nueva semiótica 
de significación y subjetividad que se constituye rostro. Así entendido, el rostro ha existido desde muy antiguo, aunque no siempre. Máquinas despóticas existieron en la antigüedad en China e India, posiblemente en otros lugares (Deleuze y Guattari 2004); pero el rostro al que refiere Deleuze coincide con su época: es el varón-cristo-platonizadosocialdemócrata-del-blanco-moderno-capitalista-neurótico. Ahí está el agenciamiento de poder específico que nos impone significancia y subjetivación al "yo mayoritario", frente al que se puede devenir una multiplicidad minoritaria (no una alteridad, nunca un "otro" que siempre remite a un "yo").

Ese es el rostro-uno que se nos impone, que debe ser superado por una rostridadmultiplicidad que exprese las intensidades subjetivas que nos recorren y en las cuales nos producimos. Deleuze mismo se refiere a tres estados respecto a la rostridad: pre-rostro de los hombres primitivos; el rostro (o rostro-cristo) de nuestra era, al cual habría que sumarle los demás adjetivos con que hemos descrito el rostro actual; $y$ las "cabezas buscadoras" (Deleuze y Guattari 2004 194). El término no es muy bello, y su traducción es compleja. Deleuze ocupa el término tetês chercheuses, que hace referencia a los cohetes provistos de una cabeza que buscan, que rastrean; es un tipo de rostridad que ha roto con el organismo, la significación y la subjetivación. De todos modos, creo que la fórmula "devenir minoritario" calza mejor con la propuesta de rostridad (o de subjetividad, si queremos plantearlo de este modo). El ensayo sobre Bene es particularmente sugerente en este punto.

Minoría designa en primer lugar un estado de hecho, o sea la situación de un grupo que, independientemente de su nombre, está excluido de la mayoría, o bien incluido, pero como una fracción subordinada en relación a un patrón de medida que establece la ley y que fija la mayoría. Se puede decir en este sentido que las mujeres, los niños, el Sur, el Tercer mundo, etc., son todavía minorías, más allá de que sean muy numerosas. Pero tomemos entonces «al pie de la letra» este primer sentido. Hay inmediatamente un segundo sentido: minoría ya no designará un estado de hecho, sino un devenir en el cual uno se compromete. Devenir mino- 
ritario, es un objetivo, y un objetivo que concierne a todo el mundo, ya que todo el mundo entra en este objetivo y en este devenir, en la medida en que cada uno construye su variación en torno a la unidad de medida despótica, y escapa, por un costado o por el otro, al sistema de poder que hacía de él una parte de la mayoría. Según este segundo sentido, es evidente que la minoría es mucho más numerosa que la mayoría [...]. Minoría designa aquí la potencia de un devenir, mientras que mayoría señala el poder o la impotencia de un estado, de una situación. Es aquí donde el teatro o el arte pueden surgir, con una función política específica. Con la condición de que minoría no represente nada de regionalista; ni tampoco de aristocrático, de estético o místico (Deleuze 2003 101).

El movimiento hacia la rostridad, hacia un modo de individuación múltiple y que escapa de la tenaza del yo, es coherente también con una postura resistente a los "poderes mayoritarios", en la medida en que se trata de sustraerse, de escapar de aquello que nos hace parte de la mayoría, o que nos vuelve organismo, es decir una carne, unos significados y una conciencia producidos por la mayoría.

\section{Final. Una carne hecha gesto}

Entre el apretado paisaje de la Lógica del sentido y sus grandes discusiones — porque las discusiones de Lógica del Sentido son, en definitiva, discusiones enormes-, llama mi atención la presencia de Joe Bousquett, el poeta paralítico. El que lleva en su propia carne una herida, tan propia, tan singular que, para Deleuze, es la forma más nítida en que el acontecimiento puede ser mostrado. Se trata de Bousquett "el estoico", el que lleva en su carne un cierto acontecimiento. Es una grieta a las tensiones del actor y del personaje. Una herida que grita en sí misma: no soy parte de ningún drama. La escenificación de los tiempos, el gran teatro del mundo, Zaratustra y el joven pastor quedan paralizados, mudos, ante ese "no sé qué" que tiene Bousquett. Bousquett el estoico. El que lleva en su propia carne un acontecimiento 
que es capaz de hacer sentido con todo otro dolor, con las bombas de Hanoi, con los gritos de Guernica, con el espanto grabado en las marcas rupestres de una caverna y con el grito de Munch. Frente a cualquier personaje, Deleuze elige a Bousquett. La razón no es muy compleja. Se trata precisamente de uno de los temas claves de El teatro y su doble de Artaud: El gritar, el gesto en su máxima expresión de descarga, por sobre toda descripción o repetición lingüística. Por sobre toda codificación. Una carne hecha gesto: "En vez de insistir en textos que se consideran definitivos y sagrados importa ante todo romper la sujeción del teatro al texto y recobrar la noción de una especie de lenguaje único a medio camino entre el gesto y el pensamiento" (Artaud 1978 101). Se trata de un gesto que va más allá de la deformación, del tartamudeo o del bilingüismo de la propia lengua (Deleuze 2003 86-91).

Estos puntos anteriores, me parece que pueden ser asumidos como algunas de esas figuras, de esos tropos que constituirían un lenguaje a medio camino entre el gesto y el pensamiento: 1 . Usar los conceptos como piezas o elementos de una puesta en escena que no puede confundirse con una representación. 2. Producirse un modo de individuación que se hace parte de un devenir minoritario. 3. Llevar en la propia carne un acontecimiento capaz de hacer sentido o comunicarse.

\section{Bibliografía}

Artaud, Antonin. El teatro y su doble. Barcelona: Edhasa, 1978.

Chevallier, Jean-Frédéric. "Por un teatro menor", Revista Colombiana de las Artes Escénicas 5 (2011): 7-19.

Chevallier, Jean-Frédéric. Deleuze et le théâtre. Paris: Ed. Les Solitaires Intempestifs, 2014.

Deleuze, Gilles. "Entrevista sobre Mil Mesetas". En Gilles Deleuze, Conversaciones. Valencia: Pre-textos, 1995.

Deleuze, Gilles. Diferencia y Repetición. Buenos Aires, Amorrortu, 2002.

Deleuze, Gilles. "Un manifiesto menos". En Carmelo Bene y Giller Deleuze, Superposiciones. Buenos Aires: Ediciones Artes del Sur, 2003. 
Deleuze, Gilles. "El método de dramatización". En Gilles Deleuze, La Isla Desierta y otros textos. Barcelona: Paidós, 2005.

Deleuze, Gilles. Lógica del sentido. Barcelona: Paidós, 2005b.

Deleuze, Gilles y Guattari, Félix. ¿Qué es la filosofía? Barcelona: Anagrama, 2000.

Deleuze, Gilles y Guattari, Félix. Mil mesetas, capitalismo y esquizofrenia. $6^{\text {a }}$ edición. Valencia: Pre-textos, 2004.

Foucault, Michel. "El sujeto y el poder". En Hubert Dreyfus y Paul Rabinow, Michel Foucault: Más allá del Estructuralismo y la Hermenéutica. México: UNAM, 1988.

Foucault, Michel. "Theatrum Philosophicum". En Michel Foucault y Gilles Deleuze, Theatrum Philosophicum, seguido de Repetición y diferencia. Barcelona: Anagrama, 1995.

Garcin-Marrou, Flore. "Gilles Deleuze, Félix Guattari and theatre. Or Philosophy and its other" TRAHIR Deuxième année, août (2011).

Klossowski, Pierre. "Cosa mi suggerisce il gioco ludico di Carmelo Bene". En Carmelo Bene, Otello, o la deficienza della donna. Milano: Feltrinelli, 1981.

Landaeta, Patricio y García Macarena. "De rostros y rastros". Konvergencias, Filosofía y Culturas en Diálogo, IV/14 Primer Cuatrimestre (2007).

Rancière, Jacques. "Política, identificación y subjetivación". En Benjamín Arditi (ed.), El Reverso de la diferencia. Caracas: Nueva Sociedad, 2000.

Sadar, Teresa. "Il teatro attraverso la filosofia di Gilles Deleuze". En Fucinemute, Non social, non network e scrittura, 2006. Disponible en http://www. fucinemute.it/2006/02/il-teatro-attraverso-la-filosofia-di-gilles-deleuze-1/

Salinas, Adán. "¿Cómo hacerse un cuerpo sin órganos? Aproximación éticopolítica a Gilles Deleuze". Hermenéutica Intercultural UCSH, Santiago de Chile 12 (2003): 297-318. 